ISSN 2079-9292

www.mdpi.com/journal/electronics

\title{
Editorial
}

\section{Acknowledgement to Reviewers of Electronics in 2014}

Electronics Editorial Office, MDPI AG, Klybeckstrasse 64, CH-4057 Basel, Switzerland

Published: 8 January 2015

The editors of Electronics would like to express their sincere gratitude to the following reviewers for assessing manuscripts in 2014:

\begin{tabular}{lll} 
Afsharimani, N. & Chen, Zhu & Haidar, Ahmad \\
Ajib, W & Choi, Jaehoon & Hannawald, Karsten \\
Alford, T.l. & Choi, Sang & Hayashi, K. \\
Althunibat, Saud & Cobelli, Claudio & He, Youjun \\
Andreoni, Giuseppe & Colodro, Carlos & Heil, Daniel P. \\
Arriola, A & Conger, Scott & Hong, Yang-ki \\
Augustyniak, Piotr & Conway, Gareth & Hu, Sanqing \\
Banos, Oresti & D'Agostino, Stefania & Huang, Ming-Chun \\
Bao, Xiulong & Darling, Seth & Hussain Abbasi, Qammer \\
Bassiouni, Mostafa & De Rossi, Danilo & Hussein, Mohamed \\
Beckert, Erik & Deen, Roshan & Irimia-Vladu, Mihai \\
Bedair, SS & DeSouza, Guilherme & Islam, MR \\
Bedogni, Luca & Dierck, A & Jacobs, Peter \\
Bergmann, Jeroen H. M. & Escalona, O J & Jong, Gwo-jia \\
Bolotov, Leonid & Espinosa, Nieves & Juciene, M \\
Boukabache, Hamza & Fiore, Marco & Jung, Byung Jun \\
Bruce, Ndibanje & Fletcher, Richard & Jung, Pyeoung-Gook \\
Cauda, Valentina & Foglia, P & Kato, Nei \\
Centurelli, F & Fukushima, Masao & Kaufmann, T \\
ČernÝ, Martin & Graditi, Giorgio & Keyhani, Ali \\
Chellappan, Vijila & Gu, Yu & Kim, Joo-Hyung \\
Chen, Fang-Chung & Guidotti, Alessandro & Kim, S \\
Chen, Jian-Zhang & Gulrez, T. & Kim, Woochul \\
\hline & &
\end{tabular}




\begin{tabular}{lll} 
Kleefeld, Andreas & Ohshima, Takeshi & Sugiura, Toshifumi \\
Kloc, Christian & Onieva, Enrique & Suh, Doug Young \\
Koch, Andreas & Pal, SK & Suh, Young Soo \\
Kondaxakis, Polychronis & Pan, David Z. & Sullivan, Charles R. \\
Koski, K & Paradiso, Rita & Takahashi, Yasutake \\
Lee, Jeong-Ik & Pérez, Joshué & Tassone, Chris \\
Li, Kwok Hung & Pernstich, Kurt & Terence, S. P. See \\
Li, Ning & Pisignano, Dario & Thotahewa, Kasun \\
Li, Xiaodong & Poon, Carmen Chung Yan & Vamvoudakis, Kyriakos G. \\
Li, Yi & Rakovich, Yury & Van Langenhove, Lieva \\
Lin, CH & Roberts, Gordon W. & Vasic, Miroslav \\
List, Emil J.W. & Roblin, Christophe & Vasilev, K. \\
Liu, Tao & Rogier, Hendrik & Vinel, Alexey \\
Loghi, Mirko & Saad, Maarouf & Vizkelethy, Gyorgy \\
Majoe, Dennis & Sakai, Masatoshi & Wang, Chien-Jen \\
Marrocchi, Assunta & Sanchez, rocio. & Wang, Lei \\
Martín, David & Sato, Soshi & Weber-Bargioni, Alexander \\
Matos, J. Nuno & Sazonov, Edward & Wei, Pei-Kuen \\
Mechau, Norman & Scatá, Marialisa & Whittow, Will \\
Melati, Rabia & Schaubroeck, David & Wietfeld, C \\
Messeguer, Roc & Scilingo, Enzo Pasquale & Xu, Chunye \\
Mishra, Amaresh & Sechilariu, Manuela & Xu, Xiaorong \\
Modelski, Jozef & Shieh, Hsin-jang & Yoon, Hargsoon \\
Mondal, TK & Shieh, Jenn-Jong & Zhao, PY \\
Monsurrò, Pietro & Song, Lingyang & Zhou, Liang \\
Naden, Aaron & Soto Campos, Ignacio & \\
Nagai, Keiji & Stojanović, Vladimir M. & \\
\hline & & \\
\hline & &
\end{tabular}

(C) 2015 by the authors; licensee MDPI, Basel, Switzerland. This article is an open access article distributed under the terms and conditions of the Creative Commons Attribution license (http://creativecommons.org/licenses/by/4.0/). 